\title{
ANUARIO DE ESTUDIOS FILOLÓGICOS
}

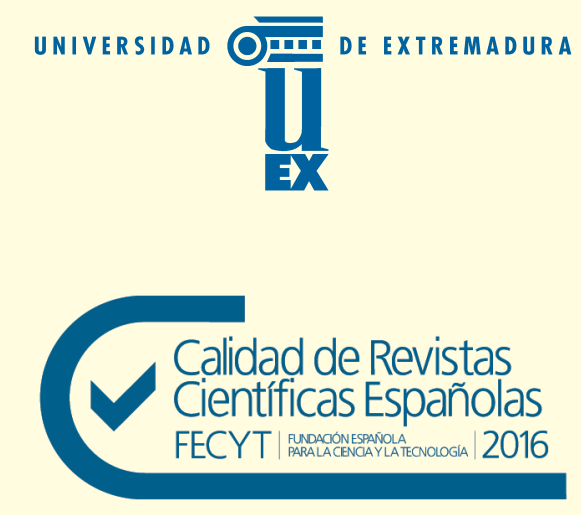

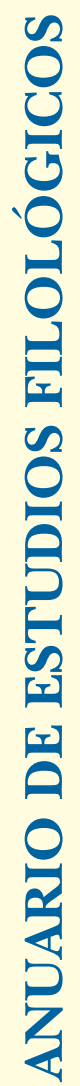

XLI/2018 
Amuario de Estudios Filológicos (AEF)

El Anuario de Estudios Filológicos es una revista de trabajos de tema filológico, de periodicidad anual, promovida por los Departamentos
de Filologia Hispanica $\mathrm{L}$ Linguístic General, Lenguas Modernas y Literaturas Comparadas, Filologia Inglesa y Ciencias de la
Antiguiedad de la Universididad de Extremadura.

DIRECCIÓN

José Manuel González Calvo (Universidad de Extremadura).

SECRETARÍA

Ramiro Gonzalez Delgado (Universidad de Extremadura).

CONSEJO DE REDACCIÓN

Pressdente: José Manuel Gonzalez Calvo (Universidiad de Extremadura).
Vocales: Jesuís Cañas Murillo (Universidad de Extremadura). Paul Danler (Universidad de Innsbruck, Austria). Salvador Gutiérrez Ordónez (Uni-

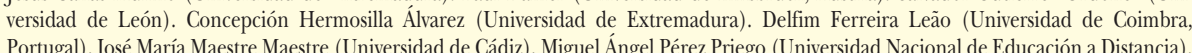

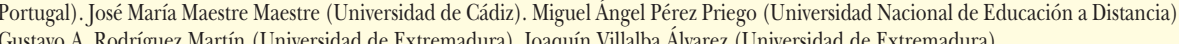
Secretario: Ramiro González Delgado (Universidad de Extremadura).

CONSEJO ASESOR EXTERNO

Ignacio Ahumada Lara (Consejo Superior de Investigaciones Científicas). Joaquín Álvarez Barrientos (Consejo Superior de Investigaciones
Gientificas). Joan Argenter Giralt (Universidad Autónoma de Barcelona). J esuis Bartolomé Gómez (Universidad del Pás Vasco). Pieddd Bohño Gennificas). Joan Argenter Giralt (Universidad Autónoma de Barcelona). Jesusis Bartolomé Gómez (Universidad del Pais Vasco). Piedad Bolaño
Donoso (Universidad de Sevilla). Manuel Casado Velarde (Universidad de Navarra). Perfecto Cuadrado Fernandez (Universidad de las Islas Baleares). Laura Dolfi (Universidad de Parma, Italia). Francisco Domínguez Matito (Universidad de La Rija). Fernando Durán López (Universidad
de Cádiz). Rosa Espinosa Elorza (Universidad de Valladolid). José Antonio Fernández Delgado (Universidad de Salamanca). Milagros Fernandez Pérez (Universidad de Santiago de Compostela). M. ${ }^{2}$ del Pilar Garcés García (Universidad de Valladolid). Ángeles Garcín Calderón (Universidac de Córdoba). Francisco García Jurado (Universidad Complutense de Madrid). Miguel Ángel García Peinado (Universidad de Córdoba). Juan Antonio Garrido Ardila (Universidad de Malta). Vicente Gonzalez Martin (Universidad de Salamanca). Augusto Guarino (Universidad de Napoles

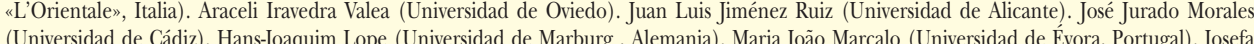
Martín García (Universidad Autónoma de Madrid). Michael Metzeltin (Universidad de Viena, Austria). José Luis Moralejo Alvarez (Universidad de Alcalá). Carmen Morenilla alalens (Universidad de Valencia). M." Azzucena Penas baanez (Universidiad Autonoma de Madrid). M.'. Cristina de Castro-

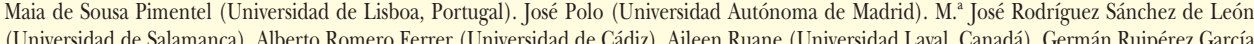
(UNED). Juan de Dios Torralbo Caballero (Universidad de Córdoba). Cristina Valdés Rodríguez (Universidad de Oviedo). Joanna Wilk-Racieska (Universidad de Silesia, Polonia). Alicia Yllera Fernández (Universidad Nacional de Educación a Distancia).

REVISIÓN DE INGLÉS: M. M. Mercedes Enríquez Aranda (Universidad de Málaga).

PRESENCIA EN BASES DE DATOS BIBLIOGRÁFICAS Y EN DIRECTORIOS Y PORTALES SOBRE INDICADORES DE CALIDAD
- SCOPUS: Elserier B.V. (NL). - CARHus Plust.
ZDB: Zeitschriftendatenbank CARHUS Plust.

- SJR: Scimago Journal \& Country Rank SUDOC.

ERIHPlus: European Reference Index

for the Human

cientificas.
Dialnet.

(a)

- DICE: Difusión y Calidad Editorial de las

Revistas Españolas.

LLBA: Linguistics and

Abstracts

$\begin{array}{lll}\text { : LBO: Linguistic Bibliography Online. } & \text { : Hispanismo (Instituto Cervantes). } \\ \text { des. las } & \text { : MLA: Modern Language Association } & \text { : Universia: Biblioteca de recursos. } \\ \text { Database. } & \text { WorldCat. } & \text { Google scholar. }\end{array}$

de revistes.

- Google scholar.

EICIÓN, SUSCRIPCIONES E INTERCAMBIO

.

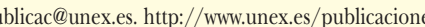

LOCALIZACIÓN DE LA REVISTA EN INTERNET

http:///anuariodestudiosfilologicos.wordpress.com/
http://www.dialnet. unirioja.es/servlet/revista?tipo_busqueda=CODIGOQ_clave_revista=111

AUTORIZACIÓN DE REPRODUCCIONES

Universidad de Extremadura.

Ninguna parte de esta revista puede ser reproducida, por ningún medio, sin el consentimiento expreso de los titulares del Copyright.

DEPOSITO LEGAL: CC.341-1978

D.S.N.: 0210-8178

FOTOCOMPOSICIÓN E IMPRESIÓN: DosGRAPHIC, S. L. - Teléf.: 91478612.5

La Universidaad de Extremadura, a los efectos previstos en el articulo 32.1, párrafo segundo del vigente TRLPI, se opone expresamente a que cualquiera de las paginas de Anuario de Estudios rihologicosos, o partes de ellas, sean unilizadas para la realizacion de resumenes de prensa.

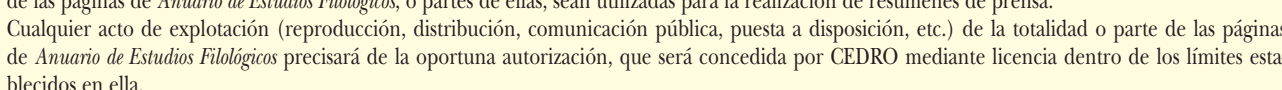




\section{ANUARIO DE ESTUDIOS FILOLÓGICOS, ISSN 0210-8178 \\ Vol. XLI, 2018}

\section{SUMARIO}

\section{ARTÍCULOS}

José Luis Arroyo Barrigüete, Hibridación genérica en ciencia ficción: análisis architextual de la trilogía Foundation de Asimov....

Jordi Bermejo Gregorio, Los falsos retratos del Teatro selecto: la canonización visual de dramaturgos barrocos por la recepción nacionalista decimonónica

Francisco Bravo de Laguna Romero, Medeas en la frontera: la tradición clásica en tres piezas teatrales latinoamericanas.

María Ángeles Díez Coronado, Tradición e innovación temática y métrica en Las Latinas o Poesías castellanas en metro latino de Esteban Manuel de Villegas

Javier Feijoo Morote, Libropueblo/Herriliburu: el proyecto editorial de Ramiro Pinilla y José Javier Rapha Bilbao (1977-1986)

EnRIQue Jiménez Ríos, Tipología de arcaísmos en el DRAE en la primera mitad del siglo XIX.

Carlos Manadé-Rodríguez y Alicia Rodríguez-Álvarez, Presencia y tratamiento de la cultura: la competencia intercultural y el humor en los manuales de ELE utilizados en Japón

Ramón Pérez Parejo, Significado y relevancia de la metapoesía de la generación del 68

Pablo Ruano San Segundo y Paloma Pizarro Seijas, Análisis de la atmósfera nebulosa a través de las palabras gramaticales en dos traducciones españolas de Heart of Darkness, de Joseph Conrad: un estudio de corpus.

Yushu YuAn, $E l$ «yo» del sujeto migrante. Análisis de «La conversión de Uei-Kuong» de Siu Kam Wen

\section{NOTAS Y DOCUMENTOS}

Olga Ivanova, A propósito de Las categorías gramaticales de Ignacio Bosque. ¿Una nueva perspectiva sobre sus relaciones y diferencias?

Ana Alicia Manso Flores, De la interjección a la adivinanza: la producción científica de Ramón Almela Pérez..... 


\section{RESEÑAS}

María José Domínguez VázQuez, Ulrich Engel y Gemma Paredes Suárez, Neue Wege zur Verbvalenz. Vol. I: Theoretische und methodologische Grundlagen.

Vol. II: Deutsch-spanisches Valenzlexikon [Alberto Bustos Plaza]

217-222

Rebeca Lázaro Niso (ed.), Corpus y bases de datos para la investigación en literatura [Alberto Escalante VARONA]

José Jurado Morales (ed.), Naturaleza de lo invisible. La poesía de Rafael Guillén [Sergio Fernández Martínez]

226-229

José Manuel González Calvo, Creatividad y expresividad en Fray Gerundio de Campazas [María Luisa Harto Trujillo]

229-232

Carmen González Vázouez (ed.), Diccionario de personajes de la comedia antigua [Álvaro IBÁÑez CHACóN]

Benjamín García Hernández y María Azucena Penas Ibáñez (eds.), Semántica latina y románica. Unidades de significado conceptual y procedimental [Laura Molina Villalba]

236-240

Gerardo Diego y Juan Larrea, Epistolario. 1916-1980 [Guadalupe Nieto Caballero]

David Huerta, La violencia en México [Diego Ernesto Parra Sánchez] ......

Jesús Sánchez lobato y Alberto Hernando García-Cervigón, Contribución al estudio de dos proyectos de gramática académica del siglo XX [María Azucena Penas Ibáñez]....

$246-251$

Cristina Pimentel y Paula Morão (coords.), A Literatura Clássica ou os Clássicos na Literatura. Presenças Clássicas nas Literaturas de Lingua Portuguesa [Marta Ramos Grané].

Elena de Lorenzo Álvarez (coord.), Ser autor en la España del siglo XVIII [Eduardo SAN José VÁzQuez]

Anejos del Anuario de Estudios Filológicos 


\section{ANUARIO DE ESTUDIOS FILOLÓGICOS, ISSN 0210-8178 \\ Vol. XLI, 2018}

\section{CONTENTS}

\section{CONTRIBUTIONS}

José Luis Arroyo Barrigüete, Generic hybridization in science fiction: architextual analysis of Asimov's Foundation trilogy.

Jordi Bermejo Gregorio, The false portraits in the Teatro selecto: the visual canonization of baroque playwrights by the 19th century nationalist reception in Spain

Francisco Bravo de Laguna Romero, Medeas in the frontier: classical tradition in three Latin American theatre plays

María Ángeles Díez Coronado, Tradition and thematic and metrical innovation in Las Latinas o Poesías castellanas en metro latino by Esteban Manuel de Villegas.

Javier Feijoo Morote, Libropueblo/Herriliburu: the publishing project of Ramiro Pinilla and José Javier Rapha Bilbao (1977-1986)

ENRIQUe JIMÉNEz Ríos, Typology of the archaisms in the Dictionary of the Royal Spanish Academy in the first half of the 19th century...

Carlos Manadé-Rodríguez y Alicia Rodríguez-Álvarez, The presence of and approach to culture: intercultural competence and humour in SFL handbooks in Japan

Ramón Pérez Parejo, Meaning and relevance of the metapoetry of the generation of ' 68 .

Pablo Ruano San Segundo y Paloma Pizarro Seijas, Grammatical words denoting vagueness in Joseph Conrad's Heart of Darkness and their translations into Spanish: a corpus approach

Yushu Yuan, The «I» of the migrant subject. Analysis of "The conversion of Uei-Kuong» by Siu Kam Wen $175-189$

\section{NOTES AND DOCUMENTS}

Olga Ivanova, On Las categorías gramaticales by Ignacio Bosque: A new perspective on their relations and differences?

Ana Alicia Manso Flores, From interjections to riddles: the scientific production of Ramón Almela Pérez. 


\section{REVIEWS}

María José Domínguez VÁzquez, Ulrich Engel y Gemma Paredes Suárez, Neue Wege zur Verbvalenz. Vol. I: Theoretische und methodologische Grundlagen.

Vol. II: Deutsch-spanisches Valenzlexikon [Alberto Bustos Plaza]

217-222

Rebeca Lázaro Niso (ed.), Corpus y bases de datos para la investigación en literatura [Alberto Escalante VARONA]

José Jurado Morales (ed.), Naturaleza de lo invisible. La poesía de Rafael Guillén [Sergio Fernández Martínez]

226-229

José Manuel González Calvo, Creatividad y expresividad en Fray Gerundio de Campazas [María Luisa Harto Trujillo]

229-232

Carmen González Vázouez (ed.), Diccionario de personajes de la comedia antigua [Álvaro IBÁÑEz CHACóN]

Benjamín García Hernández y María Azucena Penas Ibáñez (eds.), Semántica latina y románica. Unidades de significado conceptual y procedimental [Laura Molina Villalba]

236-240

Gerardo Diego y Juan Larrea, Epistolario. 1916-1980 [Guadalupe Nieto Caballero]

David Huerta, La violencia en México [Diego Ernesto Parra SÁnchez] ...... 243-245

Jesús Sánchez lobato y Alberto Hernando García-Cervigón, Contribución al estudio de dos proyectos de gramática académica del siglo XX [María Azucena Penas Ibáñez]

$246-251$

Cristina Pimentel y Paula Morão (coords.), A Literatura Clássica ou os Clássicos na Literatura. Presenças Clássicas nas Literaturas de Lingua Portuguesa [Marta Ramos Grané].

Elena de Lorenzo Álvarez (coord.), Ser autor en la España del siglo XVIII [Eduardo SAN José VÁzQuez]

Appendices of the AnUario de Estudios Filológicos. 


\title{
EL «YO» DEL SUJETO MIGRANTE. ANÁLISIS DE «LA CONVERSIÓN DE UEI-KUONG» DE SIU KAM WEN
}

\author{
YUSHU YuAN \\ China Foreign Affairs University
}

\section{Resumen}

Como escritor peruano de origen chino, Siu Kam Wen describe en el cuento «La conversión de Uei-Kuong» la vida caótica de Uei-Kuong, un peruano que se crió en China pero vuelve a vivir en Lima. Por medio del discurso múltiple del tiempo, el espacio y los narradores, Siu construye la figura del «sujeto migrante» y habla de la confusión de la identidad étnica debido a la incongruencia del «yo» cognitivo y el «mí» social. Por medio de Uei-Kuong, Siu Kam Wen logra presentar no solo el «yo individual» que sufre crisis de identidad, sino también el «yo colectivo» del hombre inmigrante chino que vive simultáneamente en dos mundos, pero condenados a pertenecer al «no lugar».

Palabras clave: Migrante, identidad, narrativa, heterogeneidad, transculturación.

\section{THE «I» OF THE MIGRANT SUBJECT. ANALYSIS OF «THE CONVERSION OF UEI-KUONG» BY SIU KAM WEN}

\begin{abstract}
As a Peruvian writer of Chinese origin, Siu Kam Wen describes in the story «The Conversion of Uei-Kuong» the chaotic life of Uei-Kuong, a Peruvian who grew up in China but returns to live in Lima. By using the multiple discourses of time, space, and narrators, Siu constructs the figure of the «migrant subject» and talks about the confusion of ethnic identity due to the incongruence of the cognitive «I» and the social «me». Through Uei-Kuong, Siu Kam Wen manages to present not only the «individual self» that suffers from identity crisis, but also the «collective self» of the Chinese male immigrants who live simultaneously in two worlds, but condemned to belong to the «no place».

Keywords: Migrant, Identity, Narrative, Heterogeneity, Transculturation.
\end{abstract}




\section{InTRODUCGión}

La pluralidad sociocultural de Latinoamérica siempre ha llamado mucho la atención de los investigadores. En el libro Contrapunteo cubano del tabaco y el azúcar Fernando Ortiz mencionó por primera vez la «transculturación», y propuso utilizar este concepto para sustituir al de «aculturación». Mientras que la aculturación se refiere al «proceso de tránsito de una cultura a otra» (Ortiz, 1983: 86), la consecuente pérdida de la cultura procedente y la adquisición de otra, la transculturación, a pesar de pérdidas inevitables del origen, se inclina por presentar los múltiples fenómenos sociales y culturales que pueden coexistir.

Posteriormente, el uruguayo Ángel Rama introdujo el concepto de transculturación a la literatura latinoamericana. Según Rama, cuando se encuentran las dos fuentes culturales distintas, habría «pérdidas, selecciones, redescubrimientos e incorporaciones», que son operaciones «concomitantes» y «se resuelven todas dentro de una reestructuración general del sistema cultural» (Rama, 2008: 40).

En el Perú, Antonio Cornejo Polar descifra la heteróclita pluralidad, una de las agendas más problemáticas de la literatura y el pensamiento de América Latina, proponiendo el concepto de la heterogeneidad. Para Cornejo Polar, la «literatura heterogénea» es «la que funciona en los bordes de sistemas culturales disonantes, a veces incompatibles entre sí, tal como se produce, de manera dramáticamente evidente, en el área andina» (Cornejo Polar, 1994: 17), es decir, las literaturas en que «se cruzan dos o más universos socio-culturales» (Cornejo Polar, 1994: 16). Gracias a este corpus, se pueden incorporar las categorías más marginales.

En los años ochenta del siglo pasado, Siu Kam Wen, un escritor peruano ${ }^{1}$ de origen chino empezó a publicar obras narrativas en las que habla de la inmigración china en Lima. En sus historias, Siu nos revela el proceso de aculturación que sufren los inmigrantes chinos de la sociedad receptora y cómo las civilizaciones oriental y occidental se mezclan en tierra peruana. Debido a su experiencia personal de varias migraciones internacionales, Siu Kam Wen se centra en construir el «sujeto migrante», que vive constantemente en dos tiempos (el pasado y el presente), dos espacios (el «allá» del pueblo natal y el «aquí» de la sociedad receptora) y dos culturas (la original y la nueva).

A pesar de que tanto Cornejo Polar como Rama proponen sus ideas bajo un contexto diferente a las que tiene Siu Kam Wen, es posible introducir

1 A pesar de que Siu Kam Wen es ciudadano estadounidense, en la entrevista con Ysla Heredia en 2017, Siu afirmó: «como escritor me siento peruano, pero íntimamente me siento chino». Siu utiliza el español como lengua principal en su creación literaria y ubica la mayoría de sus historias en Lima, por lo que se puede decir que Siu Kam Wen es un escritor peruano. 
los conceptos de transculturación y heterogeneidad en nuestro caso de estudio. Maan Lin (1997: 17) confirma en su trabajo que: «This definition of transculturation is not limited, of course, to Spanish and indigenous cultures; it can be applied to any two cultures in contact». De la misma manera, como explica Cornejo Polar, la idea de heterogeneidad goza de la flexibilidad de incluir en sí misma las categorías más marginales, como la de la etnia china.

Por lo tanto, en el presente artículo, que surge de mi investigación doctoral titulada La narrativa de Siu Kam Wen y la inmigración china al Perú, en que se estudian todas las obras narrativas de Siu Kam Wen, se realiza un análisis textual del cuento «La conversión de Uei-Kuong». Nos centramos en el discurso múltiple que utiliza el autor con el motivo de descifrar la identidad vacilante y el «yo» confuso del protagonista, un sujeto migrante chino en la narrativa peruana.

\section{Siu Kam Wen y «La conversión de Uei-Kuong»}

Siu Kam Wen, escritor peruano de origen chino, nació en la provincia Guangdong (Cantón) de China en 1951. Cuando tenía nueve años inmigró al Perú y empezó el aprendizaje del castellano, que luego se convierte en la lengua principal de su creación literaria. Vivió en Lima con su familia durante veinticinco años, antes de inmigrar a Estados Unidos, donde reside ahora.

A pesar de graduarse en la Facultad de Contabilidad de la Universidad Nacional San Marcos, Siu Kam Wen muestra un gran talento literario desde su infancia. Obtuvo varios premios de cuentos durante la época universitaria y publicó su primer trabajo, un cuento titulado «El viajero», en 1981. Su primer libro, la colección de cuentos El tramo final, salió a la luz en 1986.

En total, Siu Kam Wen tiene tres libros de cuentos: El tramo final (1986), La primera espada del imperio (1988) e Ilusionismo (1988) —este último lo publicó junto con los dos primeros en la colección Cuentos completos, edición que vamos a seguir aquí (Siu, 2004)—; cinco novelas: La estatua en el jardín (2005), El furor de mis ardores (2009), La vida no es una tómbola (2009), El verano largo (2012) y Viaje a Ítaca (2017); un ensayo redactado en inglés Deconstructing art (2004); y un drama de un acto, ¿Vino alguien después del funeral? (1991). En su entrevista en el Diario Correo, el autor comenta que es Isaac Goldemberg ${ }^{2}$ quien le inspira para escribir desde la perspectiva de un

2 Goldemberg, nacido en Chepén en 1945, es un escritor, poeta, novelista, cuentista y dramaturgo peruano. Como tiene origen judío, en sus libros describe la vida de los judíos en este país, lo que inspira a Siu Kam Wen, que también pertenece a otra minoría étnica y «extranjera». 
extranjero, en vez de imitar a Borges y su estilo fantástico, que no le resultó nada satisfactorio. Con sus historias, Siu Kam Wen lanza su mirada a la inmigración china en Perú y describe la vida verdadera del barrio chino de Lima.

$\mathrm{Su}$ primer libro, El tramo final, cuenta con nueve cuentos cortos («El deterioro», «El tramo final», «La vigilia», «El discurso», «Los compadres», «La conversión de Uei-Kuong», «En alta mar», «Historia de dos viejos»y «La doncella roja»). Con esta colección, Siu Kam Wen intenta ser casi testimonial, ofreciendo una enciclopedia social de la vida en el barrio chino de Lima en forma literaria. En el aspecto temporal, El tramo final abarca los ciento sesenta y nueve años de la inmigración china al Perú, desde la época de los culíes hasta la segunda mitad del siglo xx, época en que vivió Siu en la capital peruana. En cuanto al espacio, Siu Kam Wen revive de modo completo el barrio chino limeño, situado alrededor de la calle Capón, por medio de una descripción detallada de los restaurantes chifas, las sociedades chinas, las tiendas y las casas de importación. Lo más importante aún es que el escritor logra construir unos personajes heterogéneos de diferentes profesiones, edades y géneros, entre los cuales, Uei-Kuong, el protagonista del cuento «La conversión de Uei-Kuong», es una figura que llama mucho la atención por su experiencia rara y caótica.

El cuento «La conversión de Uei-Kuong» comienza quince días antes de la fiesta de primavera, es decir, en el Año Nuevo chino, cuando el protagonista se va a visitar a Tío Keng ${ }^{3}$, un viejo tendero chino en Lima. El tiempo inusual de este saludo al año nuevo ${ }^{4}$ parece revelar a los lectores la peculiaridad de la historia entre los dos hombres. Uei-Kuong y Tío Keng se conocen en un vuelo. El viejo tendero se siente aburrido porque no hay ningún pasajero compatriota con quien poder conversar. Sin embargo, a Tío Keng le sorprende que Uei-Kuong, un $k u e i^{5}$, es decir, «un peruano de ojos hundidos, piel cobriza y nariz pronunciada» le hable en cantonés «perfecto y fluido» (Siu, 2004: 71). Durante el viaje Uei-Kuong revela que fue adoptado por un tío chino (el cuñado de su madre) a muy corta edad. Como consecuencia se

3 En la cultura china, «tío» y «hermano» se puede utilizar delante del apellido como una forma cariñosa para referirse a una persona. Por lo tanto, en el cuento «Tío Keng», nombre del mejor amigo de Uei-Kuong, y su esposa «Tía Keng» se escriben siempre en mayúscula.

4 Este tipo de saludo, conocido como 拜年 (bài-nían en mandarín) en chino, generalmente se hace el primer día del nuevo año según el calendario lunar o dentro de los cuatro o cinco días siguientes. Nunca se adelanta porque todavía es el año «viejo».

5 鬼 (guǐ en mandarín y kuei en cantonés), que significa «demonio», es una forma negativa que utilizan los chinos en referencia a la gente extranjera. Por las diferencias físicas, por ejemplo, los ojos azules, la piel blanca, el pelo rubio o la nariz pronunciada, los chinos estaban convencidos de que los extranjeros no eran hombres, sino demonios. En el contexto de «La conversión de Uei-Kuong», la palabra kuei no tiene un sentido tan peyorativo; es igual que decir «el peruano». 
crió en el pueblo natal de este tío como cualquier niño chino: trabajaba en el arrozal, hablaba cantonés e iba a un colegio privado. Desgraciadamente, su tío murió y Uei-Kuong, con veintidós años, no tiene más remedio que recuperar su nacionalidad peruana y volver a su país de origen, al cual Tío Keng migró hace mucho tiempo.

Como no habla castellano, el protagonista se limita a vivir y buscar trabajo dentro del círculo de la colonia china. El tendero le ofrece a Uei-Kuong un puesto y, posteriormente, le presta dinero para empezar su propio negocio mientras la Tía Keng le encuentra a una buena mujer china como esposa. La nueva vida del hombre chino-peruano se organiza con éxito gracias a los Keng, pero hay una duda que nadie sabe contestar: ¿quién es Uei-Kuong? ¿un chino o un peruano?

A pesar de heredar los rasgos físicos de sus padres peruanos, culturalmente Uei-Kuong es chino. Es introvertido y se caracteriza por su timidez, rasgo que se menciona cinco veces en la descripción del personaje: «El pasajero de al lado lo estaba mirando y sonreía tímidamente» (Siu, 2004: 71); «Seguía sonriendo tímidamente, algo incómodo por haber resultado ser objeto de tanto asombro» (Siu, 2004: 72); «Respondió sin abandonar su tímida sonrisa» (Siu, 2004: 72); «Y era increíblemente tímido para un hombre de su edad» (Siu, 2004: 82); «Escuchar hablar a Uei-Kuong en cantonés fluido y verlo comportarse con timidez» (Siu, 2004: 83).

Sin duda es una cuestión de personalidad, pero generalmente los asiáticos son más tímidos que los occidentales; como comenta el mismo autor en el cuento, la timidez es «cualidad o defecto que difícilmente puede esperarse de un kuei» (Siu, 2004: 83).

Uei-Kuong tiene costumbres chinas y no logra adaptarse a los usos peruanos. Cuando acaba de llegar al Perú, su tía le espera en el aeropuerto y la anciana se muestra muy emocionada después de tanto tiempo separada de su sobrino, a quien siempre consideró como un hijo suyo. La peruana «se abalanzó sobre Uei-Kuong, cubriéndolo de besos» (Siu, 2004: 75) mientras que Uei-Kuong «no estaba acostumbrado a tan efusivas formas de exteriorizar los sentimientos, propias de temperamentos más apasionados que los de los chinos, y se quedó tieso como un trozo de leña dentro de aquellos brazos maternales, incómodo y colorado» (Siu, 2004: 75). Además, el protagonista no se divertía en las fiestas. Igual que muchos inmigrantes asiáticos por aquel entonces, «no bebía cerveza, no sabía ningún baile ni le gustaba bailar. Parecía torpe porque se conducía con una simplicidad desusada en el medio» (Siu, 2004: 82).

El sistema de valores y creencias de Uei-Kuong es también asiático: se trata de un hombre sumamente responsable en temas de trabajo y familia. Cuando 
la tía peruana insiste repetidamente en que se case, Uei-Kuong sostiene que no es el momento porque su condición económica todavía no le permite garantizar una vida segura para su futura esposa y retoños, lo que supone un gran contraste con sus primos, que se casaron muy jóvenes, pero «con la carga de una familia pendiendo sobre su cabeza como una espada de Damocles, en constantes zozobras a causa de apuros pecuniarios» (Siu, 2004: 82).

Culturalmente, no es peruano; sin embargo, debido a su apariencia física, los chinos nunca le consideran un paisano, sino un kuei. Le cuesta mucho trabajo a Tía Keng persuadir a los padres en cuanto al matrimonio de su hija con Uei-Kuong. En el círculo cerrado de la colonia, la endogamia es frecuente porque los chinos creen que los peruanos tienen demasiados vicios como para ser buenos maridos. Tía Keng prueba suerte varias veces, explicando que el protagonista es un kuei diferente y no es justo discriminarle de esa manera. Nunca tiene éxito. Se ve obligada a cambiar de estrategia y comienza a decir que Uei-Kuong es tusán ${ }^{6}$, es decir, hijo del matrimonio entre dos razas, para justificar que el protagonista de apariencia extranjera es un chino real. Sin embargo, los chinos siguen recelando ante la posibilidad de que él herede los vicios de la parte peruana.

La existencia de Uei-Kuong genera en los otros la duda acerca de su verdadera identidad, mientras que los pensamientos del mismo protagonista son muchas veces contradictorios. En el vuelo, Uei-Kuong comenta a Tío Keng que no solo habla cantonés, sino que él mismo es de esta provincia, y se presenta con el nombre chino Lau Uei-Kuong. En el aeropuerto, cuando la tía peruana le llama «Manuel», tarda mucho tiempo en acordarse de que ese es su nombre registrado, es decir, el «legítimo». Confiesa que «no sé hablar otra lengua ni comportarme de manera diferente a la de ustedes y de otros chinos» (Siu, 2004: 72), pero al mismo tiempo, es consciente de que «no soy un tusán, ni soy propiamente dicho un chino. Para ser exacto, soy lo que se llama un kuei» (ibídem).

\section{Uei-KuONG, UN «SUJETO MigRANTE»}

En el artículo «Una heterogeneidad no dialéctica: sujeto y discurso migrantes en el Perú moderno», Cornejo Polar propone el concepto de «sujeto migrante» como el resultado de su observación de la gran ola migratoria en dicho país desde las zonas rurales a las ciudades. Delimita a tal sujeto «mi-

6 Tusán es la castellanización de 土生, una palabra china que significa que una persona es nativa de algún lugar. En Perú, tusán se refiere a los hijos de inmigrantes chinos nacidos en este país latinoamericano, o a los descendientes que heredan el origen oriental de la familia. Ahora se ha ampliado el sentido de tusán y no es necesario que ambos progenitores sean chinos para ser tusánes, pero lo que sí importa es que dicha persona nazca en el Perú. 
grantes que no parecen haber perdido niveles básicos de identidad: lengua, vestido, comida, pero que al mismo tiempo - por supuesto- no pueden dejar de actuar de acuerdo a los masivos e inéditos condicionamientos que la ciudad acumula sobre ellos» (Cornejo Polar, 1996: 838). Al estudiar la tradición literaria del sujeto migrante en el Perú, formula la hipótesis de lo descentrado que es el discurso a la hora de construir la identidad borrosa y vacilante de dicho grupo: estos sujetos migrantes viven constantemente en dos líneas de tiempo —el hoy y el ayer — y dos lugares —el aquí y el allá-. A pesar de que su estudio se centra en los serranos que se trasladan a vivir en las ciudades, en nuestro caso, el protagonista de «La conversión de UeiKuong» comparte con estos sujetos migrantes peruanos la experiencia de vivir simultáneamente en dos mundos. Al mismo tiempo, en su cuento Siu también utiliza el discurso múltiple en cuanto al narrador para construir la figura borrosa de Uei-Kuong, un hombre que «inmigra» al Perú.

En primer lugar, en «La conversión de Uei-Kuong», Siu Kam Wen no sigue el tiempo cronológico, sino que rompe el orden normal de sucesión de acciones. El cuento empieza en el presente: Uei-Kuong visita a Tío Keng en su tienda antes del Año Nuevo chino. Al ver a los parroquianos «boquiabiertos» (Siu, 2004: 70) por la conversación en cantonés entre los dos, Tío Keng se acuerda inmediatamente de la sorpresa que sintió al enterarse de la vida que había llevado este hombre chino-peruano en el vuelo a Lima. Cuando los dos se sientan en la trastienda a tomar el té, Tío Keng sigue observando a su amigo porque, durante todo el tiempo que llevaban trabajando juntos, la existencia del protagonista siempre generaba en él un sentimiento complicado de amor-odio. Uei-Kuong no ha cambiado mucho, concluye el viejo tendero a medida que recuerda los días pasados, cuando el protagonista le pidió prestado dinero para iniciar su propio negocio. Al final, el cuento regresa al presente: Uei-Kuong habla de su hijo, cuestión que proyecta su historia hacia el futuro.

La historia se desarrolla con el flujo de pensamiento de la voz narradora (Tío Keng). Como consecuencia, se mezclan el hoy y el ayer, que aparecen por turnos en el cuento. El escritor destruye intencionalmente la historia como conjunto y la presenta en forma de fragmentos desordenados. De este modo, exige lectores dinámicos que ordenen las piezas según el hilo conductor que deja el autor; con este motivo en mente, Siu Kam Wen también deja caer una pequeña pista con la que marca cada cambio temporal: la primera palabra de cada sección se escribe totalmente en mayúsculas:

QUINCE días antes del Año Nuevo - 1. Presente

EL CAMBIO de avión fue en el aeropuerto de San Francisco - 1. Pasado DESPUÉS de estrecharles la mano al yerno y a la hija del Tío Keng 2. Presente 
VARIAS semanas después de su regreso a Lima - 2. Pasado UEI-KUONG saboreaba su té con verdadera fruición - 3. Presente HABÍA pasado Uei-Kuong cuatro años en forma ininterrumpida en la tienda del Tío Keng - 3. Pasado

¿CÓMO va tu chacra? - 4. Presente

En segundo lugar, los dos tiempos también se relacionan con los dos espacios en que vive el sujeto migrante: el aquí y el allá, es decir, el punto de partida — el pueblo natal en Cantón-y el destino — Lima- La memoria de la zona rural donde Uei-Kuong pasaba la infancia siempre se vincula con la naturaleza y los días libres de preocupaciones:

A los cinco años fue puesto en una escuelita particular donde le hicieron aprender el Sam Chi Ken, un libro de palabras elementales agrupadas en «versos» de tres ideogramas cada uno, y le enseñaron a escribir con pinceles. En otoño se iba a los cerros a volar comentas y en verano a nadar en los riachuelos. Se subía a los árboles para robarles los huevecillos a los pajaritos, cazaba a los grillos para enfrentarlos en duelos contra los de otros chicos, y de noche iba a los arrozales a atrapar luciérnagas (Siu, 2004: 73).

Se percibe un tono nostálgico y, para el protagonista, el pueblo supone un paraíso perdido al que nunca más podrá volver durante el resto de su vida. Este tipo de retórica, como señala Cornejo, existe en la tradición literaria del Perú. Por ejemplo, en «Idilio muerto», César Vallejo construye una oposición entre «la plenitud del ayer rural de la andina y dulce Rita, y la deficiencia del presente urbano en que sufre y se enajena el poeta». Explica Cornejo Polar (1996: 839):

Es importante subrayar que desde muy antiguo y hasta hoy existe algo así como una retórica de la migración que pone énfasis en sentimientos de desgarramiento y nostalgia y que normalmente comprende el punto de llegada - la ciudad- como un espacio hostil, aunque de algún modo fascinante o simplemente necesario, a la vez que sitúa en el origen campesino una positividad casi sin fisuras, con frecuencia vinculada a una naturaleza que es señal de plenitud y signo de identidades primordiales.

Es lógico que la añoranza del «allá rural» surja generalmente de la hostilidad del «aquí urbano», pero la nostalgia y el éxito en la nueva vida no son dos elementos que no puedan coexistir simultáneamente en una persona: Uei-Kuong vive bien en Lima, pero su corazón pertenece al campo. A pesar de la influencia de la capital, el protagonista nunca cambia. Es siempre «fuerte, infatigable, empeñoso, de trato fácil y agradable» (Siu, 2004: 78) y se notan claramente en él las huellas rurales, incluso después de veinte años residiendo en una ciudad. 
La resistencia de la identidad original campesina de los inmigrantes también se puede interpretar desde una perspectiva económica. De acuerdo con Cornejo Polar (1996: 840):

Es inexacto imaginar que la migración opera como fuerza imbatible y todopoderosa que reconstruye desde sus raíces la identidad del migrante campesino, convirtiéndolo, por ejemplo, en protagonista de la «larga marcha» - supuestamente casi siempre exitosa- hacia la propiedad privada y el capitalismo (De Soto, 1986), entre otras muchas razones porque el migrante tiende a repetir en la ciudad modos de producción y de relaciones sociales, como la reciprocidad, la operatividad económica de la familia ampliada o el simple padrinazgo, que difícilmente se incorporan a las normas del capitalismo moderno.

No es nuestra intención analizar si Uei-Kuong llega o no a incorporarse al sistema capitalista del Perú, porque el autor no nos describe claramente la profesión de su protagonista. Por las conversaciones, solo sabemos que, después de cuatro años, el hombre renunció a su puesto en la tienda de Tío Keng e inició su propio negocio. Ahora tiene una chacra y tierra de cultivo. Por lo tanto, se supone que Uei-Kuong repite el modelo de producción agrícola e intenta mantener dentro de su hogar el sistema tradicional de las familias chinas. Está preocupado por su hijo "peruanizado», que no quiere hablar cantonés y no tiene respeto hacia los padres. Para describir su desesperación, Uei-Kuong recurre a unas metáforas que revelan que su forma de pensamiento es todavía la de un campesino: «suspirando, sintiéndose más que nunca impotente. Solía sentir lo mismo cuando algunas veces, ya fuera en Pun-yi o en Chincha, no había lluvia por meses y las tierras labradas se secaban y se cuarteaban ante sus ojos, sin que él pudiera hace nada para remediar la situación» (Siu, 2004: 85).

En tercer lugar, en este cuento Siu Kam Wen utiliza una voz narrativa múltiple. Siu Kam Wen, el autor, nos narra la historia de la vida de Uei-Kuong, el protagonista, desde la perspectiva de su amigo Tío Keng, el testigo. Curiosamente, la perspectiva de Tío Keng, la voz narradora y testigo de este cuento, es también vacilante. Cornejo Polar (1996: 843) cita a Juan Biondi y Eduardo Zapata por la anécdota de un «cómico ambulante»: primero, el orador se declaró un serrano, pero luego se identificó como un limeño criollo. Posteriormente volvió a enfatizar el orgullo que sentía como provinciano: «si tú eres provinciano nunca niegues a tu tierra. Yo vivo orgulloso como serrano que soy, serrano a mucha honra, serranazo» (Biondi y Zapata, 1994: 448).

Es posible que esta sea la técnica adoptada por el orador para poder agradar al público, pero no se puede negar que su discurso sufre constantes cambios, debido a que vive al mismo tiempo en dos mundos. A pesar de 
ser el narrador de una historia ajena, la perspectiva de Tío Keng, la de un inmigrante, no es única frente al otro sujeto migratorio. Generalmente, el viejo tendero tiene el tono típico de un chino que, al observar a su amigo extranjero, intenta comparar las similitudes y las diferencias entre peruanos y chinos. No obstante, hay ocasiones en que Tío Keng deja de ser un chino frente al novato en un país desconocido. Por ejemplo, cuando ve a la tía abrazando a Uei-Kuong en el aeropuerto, Tío Keng describe: «se quedó tieso como un trozo de leña dentro de aquellos brazos maternales, incómodo y colorado» (Siu, 2004: 76). La identidad se cambia entre los dos hombres: Tío Keng parece un peruano frente a Uei-Kuong, un chino torpe que no acostumbra a expresar públicamente sus emociones de modo tan abierto.

Por lo tanto, debido a la yuxtaposición de tiempo, de espacio y de diferentes perspectivas del narrador, en «La conversión de Uei-Kuong» Siu Kam Wen, al igual que en la literatura del sujeto migrante serrano en el Perú, construye una figura borrosa. Intenta encontrar un equilibrio entre la cultura antigua y la nueva de la sociedad receptora. Como resultado, este sujeto migrante vacila constantemente entre los dos mundos.

\section{LA CONFUSIÓN DE LA IDENTIDAD ÉTNICA: EL «YO» COGNITIVO Y EL «MÍ» SOCIAL}

En «La conversión de Uei-Kuong», Siu Kam Wen logra construir una figura que vacila siempre entre los dos mundos, preguntando si él es chino o peruano. La existencia de este hombre raro genera la confusión de la identidad étnica en los otros, como Tío Keng, y en él mismo.

Al estudiar los inmigrantes adolescentes en España, Elena Briones (2010) señala que la identidad étnica se refiere al significado subjetivo de la propia etnicidad y los sentimientos que uno mantiene hacia el propio grupo étnico. En realidad, existen otros términos similares como la identidad cultural, la social y la racial, pero, en este caso, la identidad étnica que delimitan Phinney y Ong (2007) es la más adecuada: en primer lugar, la identidad social y la cultural resultan conceptos más amplios de lo que aparentan: la identidad social engloba la étnica, mientras que la cultural abarca más elementos según explica Briones (2010: 45):

En cuanto a la delimitación de la identidad cultural y la identidad étnica, nos encontramos con que pueden ser conceptualizadas de manera similar, aunque Jensen [2003] y Phinney et al. [2001] consideran que la identidad cultural es más amplia y envolvente. Es decir, estas autoras definen la identidad étnica como el significado subjetivo de la propia etnicidad y los sentimientos que uno mantiene hacia el propio grupo étnico [Phinney, 1992; Roberts et al. 1999], mientras que la identidad cultural se refiere a los valores, ideales 
y creencias específicos adoptados por un grupo cultural dado, así como al sentimiento de pertenencia hacia el propio grupo [Jensen, 2003].

En segundo lugar, hay diferencias entre la identidad étnica y la racial porque la segunda corresponde a la raza y al racismo:

Quintana et al. [2006] señalaron la especial importancia de las aproximaciones que diferencian raza y etnicidad de cultura en el desarrollo de los jóvenes. El término raza se refiere a su significado socialmente construido [Smedley y Smedley, 2005], en el cual las diferencias entre los grupos raciales son percibidas como inmutables, debido a la creencia de que las diferencias raciales están basadas en características genéticas y biológicas. Por tanto, las connotaciones socialmente construidas del término raza son las que le diferencian del estatus étnico o cultural; Phinney y Ong [2007] mantienen que el estudio de la identidad racial ha surgido como respuesta al racismo, y que en la medición de la identidad racial se han valorado las experiencias relativas a la internalización del racismo. Por el contrario, la identidad étnica ha sido estudiada en referencia al sentimiento de pertenencia a un grupo étnico, es decir, a un grupo definido por la propia herencia cultural, incluyendo valores, tradiciones e idiomas (Briones, 2010: 44).

Por eso, la identidad étnica es el concepto más adecuado, porque nos centramos en las diferencias de las dos culturas (la china y la peruana), en aspectos como el idioma, la tradición, la costumbre, entre otras cosas, y el sentido de pertenencia de un sujeto migrante hacia cierto grupo.

La identidad étnica está compuesta por varios elementos. Investigadores como Darío Páez y José Luis González (2000) opinan que la identidad étnica incluye facetas tan básicas como la autocategorización, las actitudes y los sentimientos hacia el propio grupo, el conocimiento de los valores y las tradiciones, las actitudes, el dominio del idioma y la implicación con el resto de miembros del grupo y sus prácticas, así como la evaluación de estas prácticas, entre otras cosas. Pero, según Phinney y Kohatsu (1997), los dos factores fundamentales son el sistema cognitivo y el afectivo. De acuerdo con Briones (2010: 46), la parte cold, de lo cognitivo, se refiere a «el grado en el cual se explora la importancia de pertenecer a uno o más grupos» mientras que lo hot de lo afectivo es «el grado en el que la gente siente conectar con su grupo o en la medida en la que afirman ser miembros de un grupo».

La famosa teoría del Self de George Herbert Mead (1982) nos ofrece una posible forma de interpretar mejor la coexistencia de los sistemas cognitivo y afectivo en la identidad étnica. De acuerdo con este psicólogo estadounidense, en la personalidad de un individuo existen al mismo tiempo el «mí», las actitudes organizadas por los otros y adoptadas por el individuo, y el «yo», la reacción de dicha persona frente a las opiniones ajenas; es decir, las 
opiniones de otra gente afectan a nuestros comportamientos e ideas, pero la reacción tarda más tiempo en llevarse a cabo. Mead (1982: 203) explica la función del «yo»y el «mí» con el ejemplo de un juego de fútbol:

Ahora bien, en la medida en que el individuo despierta en sí las actitudes de los otros, surge un grupo de reacciones organizadas. Y el que logre tener conciencia de sí se debe a la capacidad del individuo para adoptar las actitudes de esos otros en la medida en que estos pueden ser organizados. La adopción de todas esas series de actitudes organizadas le proporciona su «mí»; esa es la persona de la cual tiene conciencia. Puede lanzar la pelota a algún otro miembro gracias a la exigencia que le presentan otros miembros del equipo. Esa es la persona que existe inmediatamente para él en su conciencia. Tiene las actitudes de cualquier acto de él, y ha asumido la responsabilidad de la situación. Pues bien, la presencia de esas series de actitudes organizadas constituye ese «mí» al cual reacciona como un «yo». Pero ni él ni ningún otro sabe cuál será dicha reacción. Quizás haga una jugada brillante o cometa un error. La reacción a esa situación, tal como aparece en su experiencia inmediata, es incierta, y ello es lo que constituye el «yo».

Por lo tanto llegamos a la conclusión de que el «yo» es consciente, mientras el «mí» es social; la relación entre ambos, según Mead (1982: 205), es «algo que reacciona a una situación social que se encuentra dentro de la experiencia del individuo». Como hombres nacidos con «cierta nacionalidad, ubicados en cierto punto geográfico, con tales y cuales relaciones familiares y tales y cuales relaciones políticas» (Mead, 1982: 209), todos estos factores constituyen el «mí» de este individuo, es decir, cómo es él o ella para los otros.

La actitud borrosa de Tío Keng frente a este hombre chino-peruano tan complicado explica muy bien el «mí», es decir, las opiniones de los otros según lo que explica Mead. Para el viejo tendero, los kueis no son de fiar porque a los pocos empleados peruanos que había tenido «tarde o temprano los había sorprendido robando el dinero o escamoteando las mercancías de la tienda» (Siu, 2004: 76), pero eso nunca le ha ocurrido con trabajadores compatriotas. En realidad, no se trata de que los chinos sean más decentes, sino que se preocupan más por su honor que los peruanos: como se limitan a trabajar en el círculo cerrado del barrio chino, perder el «buen nombre» es igual que un suicidio. Por lo tanto, lo que siente el viejo tendero hacia Uei-Kuong se ve influenciado por este juicio básico. Cuando el protagonista habla en cantonés, el tendero es capaz de persuadirse de que Uei-Kuong es chino y le trata con toda la confianza y cariño que tiene a su compatriota; sin embargo, una vez que Uei-Kuong queda en silencio o intenta expresarse en el escaso español que ha aprendido, a Tío Keng le asaltan «temores» y «recelos repentinos» (Siu, 2004: 78), porque con la cara occidental de Uei- 
Kuong, el viejo tendero no puede convencerse de que su mejor amigo no sea un peruano.

A través de la absorbente descripción de las vacilaciones y contradicciones de Tío Keng, Siu Kam Wen nos ofrece una interpretación literaria del «mí chino»y el «mí peruano» del sujeto migrante en la opinión de otra persona. Los dos «mis» de Uei-Kuong aparecen dependiendo de la lengua: cuando habla cantonés, el protagonista presenta ante otros el «mí chino»; al quedarse callado, la apariencia física sobrepasa a la cultura y en el protagonista forma el «mí peruano». La coexistencia del «mí chino»y el «mí peruano» agudiza la confusión que tiene Uei-Kuong de a qué mundo pertenece él en realidad. Las actitudes contradictorias de los otros influyen en la función normal del «yo» cognitivo y al final le condena a pertenecer al «no lugar»: el protagonista no se considera chino porque en realidad no lo es, pero tampoco se siente peruano porque no le gusta.

\section{Conclusión}

En cierto sentido, la creación literaria es como un espejo, porque a través del proceso de redacción, los escritores pueden conocerse mejor a sí mismos, como un narrador de autorreflexión: escribir le da a Siu Kam Wen la oportunidad de explorar lo que se esconde en lo más profundo de su alma.

En las obras autobiográficas y semiautobiográficas, Siu abre las heridas del pasado con el motivo de revelar sus sensaciones más íntimas. Por medio de este proceso de reflexión-exploración-redacción, Siu Kam Wen logra descifrar su problema: la incongruencia de los sistemas cognitivos y afectivos como un sujeto migrante.

En las historias que navegan entre la ficción y la realidad, los personajes, especialmente los masculinos, comparten con su autor la confusión de la identidad. En el caso de «La conversión de Uei-Kuong», el protagonista Uei-Kuong «inmigra» al Perú con un sistema chino ya establecido, pero en el nuevo destino se ve obligado a incorporarse a la cultura de los «otros» para adaptarse a una nueva vida. Siempre intenta encontrar un equilibrio entre los dos mundos. No obstante, los «otros» forman juicios igualmente distintos sobre qué es Uei-Kuong y cómo es él. Así, este hombre tiene al mismo tiempo un «mí chino»y un «mí peruano». Frente a los «mis» contradictorios, el «yo» no es capaz de llegar a una definición única y conforme de sí mismo.

Para construir la figura vacilante y complicada de Uei-Kuong, el escritor se aprovecha de múltiples voces narradoras: una voz de autor (Siu Kam Wen) que estructura la historia, una voz de testigo (Tío Keng) que ofrece una observación con el fin de ampliar la extensión del acontecimiento, y una voz de actor (Uei-Kuong) que aporta más dramatismo. Gracias a los múltiples 
niveles de narradores, el autor logra presentar los acontecimientos de un modo más completo, explicar claramente los dilemas que tienen los hombres chinos y explorar el mundo interior de sus personajes. Además, para presentar la vida caótica, Siu Kam Wen aplica un discurso doble del tiempo - el presente y el pasado- y del espacio —el aquí y el allá-. Con estas técnicas de yuxtaposición, Siu logra mezclar la historia oriental con la técnica literaria latinoamericana y crear un sujeto migrante chino en la narrativa peruana.

Además de Héctor/Siu, la figura (semi)autobiográfica y Uei-Kuong, Siu Kam Wen también construye una serie de personajes masculinos chinos cuya identidad está en constante crisis. La duda de la identidad étnica deja de ser un caso privado de una persona y se hace un problema colectivo de la comunidad. Basándose en la confusión que comparte el protagonista chinoperuano con su grupo, Siu amplía el concepto del «yo»: no solo hay un «yo individual» de Siu Kam Wen o Uei-Kuong, sino también un «yo colectivo» de los inmigrantes chinos, especialmente los varones.

Por medio de la autorreflexión, autoexploración y las técnicas de yuxtaposición, en las obras narrativas representadas por el cuento «La conversión de Uei-Kuong», Siu Kam Wen crea un «yo» complicado del sujeto migrante y vincula las figuras masculinas con la confusión y la identidad en crisis. En el sentido individual, por medio de Uei-Kuong, Siu Kam Wen explora el «yo» como una persona que realiza una larga búsqueda de las respuestas en la circunstancia caótica de la migración; en el sentido colectivo, es el «yo» como un miembro de la inmigración china al Perú, que reflexiona constantemente sobre la relación que mantiene este grupo con los dos mundos que coexisten en su vida. Por medio de este «yo colectivo», Siu logra resumir las características de Uei-Kuong y otros personajes masculinos como inmigrantes y revelar su mundo psicológico como chinos.

\section{BibLIOGRAFÍA}

Biond, J. y Zapata, E. (1994): Representación oral en las calles de Lima. Lima, Universidad de Lima.

Briones PÉrez, E. (2010): La aculturación de los adolescentes inmigrantes en España: aproximación teórica y empirica a su identidad cultural y adaptación psicosocial. Salamanca, Universidad de Salamanca.

Cornejo Polar, A. (1996): «Una heterogeneidad no dialéctica: sujeto y discurso migrantes en Perú moderno». Revista Iberoamérica, 62 (176-177), págs. 837-844.

Jensen, L.A. (2003): «Coming of Age in a Multicultural World: Globalization and Adolescent Cultural Identity Formation». Applied Developmental Science 7, págs. 189-196.

Lin, M. (1997): Writers of Chinese Diaspora: Siu Kam Wen in Peru. Tesis doctoral: Education and Teacher College, Columbia University. 
Mead, G.H. (1982): Espiritu, persona y sociedad: desde el punto de vista del conductismo social (trad. G. Germani). Barcelona, Paidós.

Ortiz Fernández, F. (1983): Contrapunteo cubano del tabaco y de azúcar. La Habana, Editorial de Ciencias Sociales.

Páez, D. y González, J.L. (2000): «Culture and Social Psychology». Psicothema, 12, págs. 6-15.

Phinney, J. (1992): «The Multigroup Ethnic Identity Measure: A New Scale for Use with Diverse Groups». Journal of Adolescent Research, 7, págs. 156-176.

Phinney, J. et al. (2001): «Ethnic Identity, Immigration, and Well-being: An International Perspective». Journal of Social Issues, 57, págs. 493-510.

Phinney, J. y Kohatsu, E. (1997): «Ethnic and Racial Identity Development and Mental Health». En Schulenberg, J. et al. (eds.): Health risks and development transitions during adolescence. New York, Cambridge University Press, págs. 420-443.

Phinney, J. y Ong, A. (2007): «Conceptualization and Measurement of Ethnic Identity: Current Status and Future Directions». Journal of Counseling Psychology, 54, págs. 271-281.

Quintana, S. et al. (2006): «Race, Ethnicity, and Culture in Child Development: Contemporary Research and Future Directions». Child Development, 77, págs. 1129-1141.

Rama, A. (2008): Transculturación narrativa en América Latina. Buenos Aires, El Andariego.

Roberts, R. et al. (1999): «The Structure of Ethnic Identity in Young Adolescents from Diverse Ethnocultural Groups». Journal of Early Adolescence, 19, págs. 301-322.

Siu, K.W. (2004): Cuentos completos. Morrisville, Lulu Inc.

Smedley, A. y Smedley, B. (2005): «Race as Biology is Fiction, Racism as a Social Problem is Real: Anthropological and Historical Perspectives on the Social Construction of Race». American Psychologist, 60, págs. 16-26. 